\title{
Computer-aided Design and Computer-aided Manufacturing Restorations with Minimal Invasive Approaches
}

\author{
Emine Mustafaoğlư ${ }^{1}$, Özge Ünal ${ }^{2}$, Bora Bağış ${ }^{3}$
}

\begin{abstract}
Implant-supported crowns, full-coverage fixed dental prostheses and inlay-retained fixed partial prostheses are treatment methods typically used for the rehabilitation of a single missing posterior tooth. With the increasing emphasis on conservative treatment in dentistry, inlayretained adhesive restorations are becoming popular. With the progress of computer-aided design and computer-aided manufacturing (CAD/ CAM) technology, ceramic restorations in the posterior region can be fabricated with sufficient esthetic and mechanical properties. This case report illustrates an alternative approach to the rehabilitation of narrow edentulous spaces with ceramic materials by CAD/CAM technique with minimally invasive approaches.
\end{abstract}

Keywords: All-ceramic restorations, CAD/CAM, Minimal invasive.

International Journal of Prosthodontics and Restorative Dentistry (2020): 10.5005/jp-journals-10019-1258

\section{BACKGROUND}

With the development of computer-aided design and manufacturing (CAD/CAM) methods, dental restorations might be fabricated with different designs in shorter times. The introduction of dental ceramics with increased mechanical strength and the ability of adhesive technology to bond well to these materials have increased the interest of dentists in making minimally invasive restorations. Rehabilitation of the edentulous space with minimal tooth removal is promising for both dentists and patients. There are many treatment options for large diastemas and single missing teeth in the posterior region due to different reasons. The health and economic status of the patient, size of the missing area, and position of the adjacent teeth can influence the selection of the treatment methods.

Although implant-supported restorations have gained popularity when treating a single missing tooth, sometimes this treatment type is not preferred among the patients because of its high cost, length of treatment, and surgical intervention. ${ }^{1}$ Other important contraindications of dental implant treatment are the narrow edentulous space and the proximity of the implant site to the anatomical risk areas. When a tooth is missing, the adjacent teeth may tip into the edentulous space, so the adjacent teeth may need to be restored also for occlusal rehabilitation. In this case report, alternative treatment protocols were applied in the presence of narrow posterior edentulous spaces.

In cases where dental implant treatment cannot be performed, the most commonly used treatment option is metal-based fixed dental prostheses which need large preparations of adjacent teeth. ${ }^{2}$ Both the need for a significant amount of tooth preparation and the problems associated with the long-term periodontal restorative inter-relationship, as well as allergy conditions, can often exclude patients from dentist treatment. Over the years, there has been a growing interest in inlay-supported bridges, which are a less invasive treatment option, compared to a three-unit fixed dental prosthesis which results in significant loss of dental structure in the treatments. ${ }^{3}$

In the past, gold alloys have been preferred as the base material in the construction of inlay-retained fixed dental prostheses. However, increasing esthetic needs in dentistry have led to a focus
1,2Department of Prosthodontics, Faculty of Dentistry, Izmir Katip Çelebi University, İzmir, Turkey

${ }^{3}$ Department of Prosthodontics, Faculty of Dentistry, Izmir Democracy University, Izmir, Turkey

Corresponding Author: Bora Bağış, Department of Prosthodontics, Faculty of Dentistry, Izmir Democracy University, İzmir, Turkey, Phone: +90-532-6804656, e-mail: bbagis@yahoo.com

How to cite this article: Mustafaoğlu E, Ünal Ö, Bağış B. Computer-aided Design and Computer-aided Manufacturing Restorations with Minimal Invasive Approaches. Int J Prosthodont Restor Dent 2020;10(1):39-43.

Source of support: Nil

Conflict of interest: None

on indirect metal-free restorations, which have become more popular than metal ceramic prostheses. ${ }^{4}$

It is foreseen that the use of ceramics with CAD/CAM technology in block form will also show advantages in terms of their mechanical strength over ceramics produced by other fabrication methods. ${ }^{5}$

Zirconium oxide ceramics are accepted from the all-ceramic restorations group with the best mechanical strength among ceramics. ${ }^{6}$ For these reasons, monolithic zirconia ceramics are perhaps the first all-ceramic option to come to mind in recent years for the rehabilitation of missing posterior teeth. Although problems have been reported in adhesion to zirconia, ${ }^{7}$ it is also known to have achieved sufficient bonding strength by the use of various silanes and resin cements containing a phosphate monomer [10-methacryloyloxydecyl dihydrogen phosphate (MDP)]..$^{8-10}$

In this case report, ceramic restorations are reported in a patient with narrow posterior edentulous spaces by CAD/CAM technique with a minimally invasive approach.

\section{Case Description}

A 32-year-old female patient applied to the prosthetic dental clinic with the request of rehabilitation of newly extracted teeth 12,14 , and 15 and teeth 45 and 36 that were lost previously. Poorly fitting crown restorations were observed in teeth 11 and 16 . For

() The Author(s). 2020 Open Access This article is distributed under the terms of the Creative Commons Attribution 4.0 International License (https://creativecommons. org/licenses/by-nc/4.0/), which permits unrestricted use, distribution, and non-commercial reproduction in any medium, provided you give appropriate credit to the original author(s) and the source, provide a link to the Creative Commons license, and indicate if changes were made. The Creative Commons Public Domain Dedication waiver (http://creativecommons.org/publicdomain/zero/1.0/) applies to the data made available in this article, unless otherwise stated. 
the rehabilitation of the upper jaw, it was decided to construct a zirconia-based ceramic bridge with the support of teeth 11,13 , and 16 , upon patient request. Tipping and migration of neighboring teeth were observed due to the lack of teeth 45 and 36 for a long time, as mentioned by the patient. After clinical and radiological examinations, oral hygiene of the patient was found to be good and periodontal tissues were healthy. There were composite restorations in the distal adjacent teeth 46 and 37 . Both teeth were inclined mesially and narrow edentulous spaces were determined (Fig. 1). Implant treatment was not a possible option because of
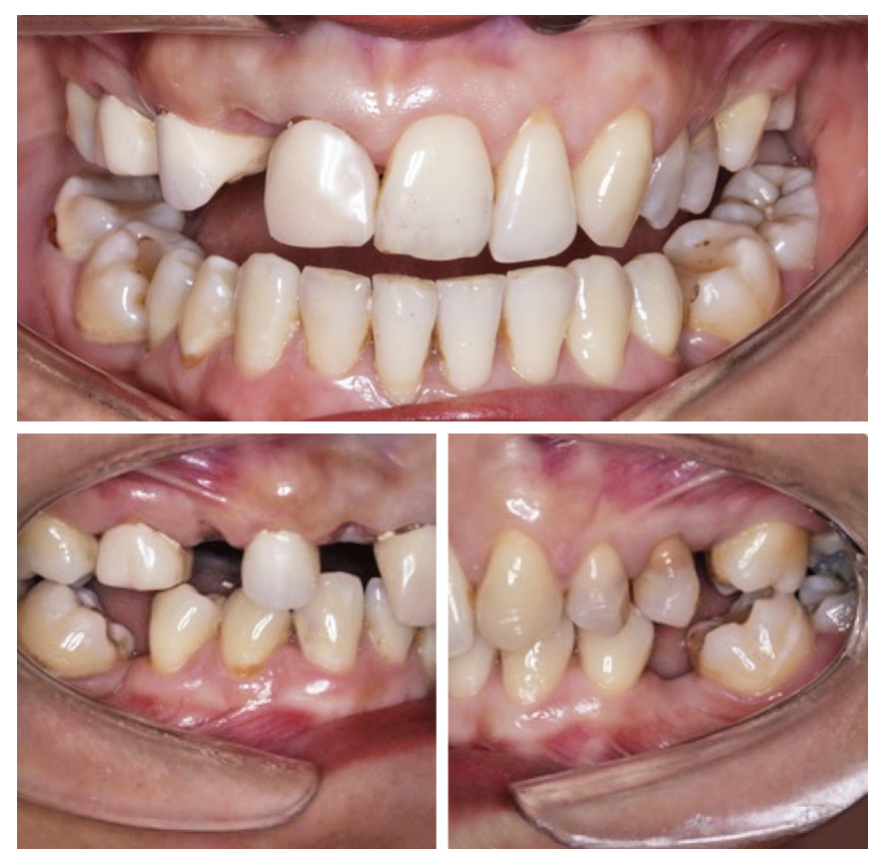

Fig. 1: Intraoral views of the patient before the treatment the limited area. The patient did not choose possible orthodontic treatment because of the length and cost of the treatment. After an evaluation of the treatment alternatives with the patient, an onlay restoration was planned with feldspathic ceramic (Vita Mark II; Vita Zahnfabrik, Bad Säckingen, Germany) material for tooth 46 area and onlay-retained pontic design fixed partial denture with monolithic zirconia material (DD cubeX ${ }^{2}$ multilayer-cubic zirconia system, 5Y-TZP, and A3; Dental Direkt, Spenge, Germany) by using $\mathrm{CAD} / \mathrm{CAM}$ technique.

After removing the composite restorations, partial tooth preparations were performed using a minimum of $1.5 \mathrm{~mm}$ depth thickness to the corresponding arch and cavity walls flaring to 5-15. Digital impressions (Omnicam, Sirona, Bensheim, Germany) were taken. Then, the restorations were designed in CAD/CAM system (Fig. 2).

In order to overcome the color harmony problems in the monolithic zirconia material, a window-shaped design was formed on the buccal surface of the restoration in tooth 37 (with a veneerstyle design), and monolithic feldspathic ceramic block (A3 and Vita Mark II) was used in tooth 47.

In the fabrication of tooth 37 restoration, the milling process was performed by using CAD/CAM technology. Following the $A 3$ coloring process of the restoration, sinterization was performed at $1,500^{\circ} \mathrm{C}$ for 2 hours. After sintering, porcelain was added to the veneer area on the buccal side of the restoration. Other surfaces were finished with mechanical polish. Sandblasting $\left(50 \mu \mathrm{m}, \mathrm{Al}_{2} \mathrm{O}_{3}\right)$ was applied to the internal surfaces of the zirconia restoration. Then, these surfaces were treated with primer (Clearfil Ceramic Primer Plus; Kuraray Noritake Dental, Inc., Osaka, Japan) containing the phosphate monomer MDP and finally luted with dual-cure resin cement (Panavia F 2.0 Light; Kuraray Noritake Dental, Inc.). For the feldspathic ceramic restoration, etching was performed with hydrofluoric acid (Porcelain Etchant-9.5\% buffered hydrofluoric acid gel) and then silane (Porcelain Silane; B.J.M. Laboratories Ltd, Israel),
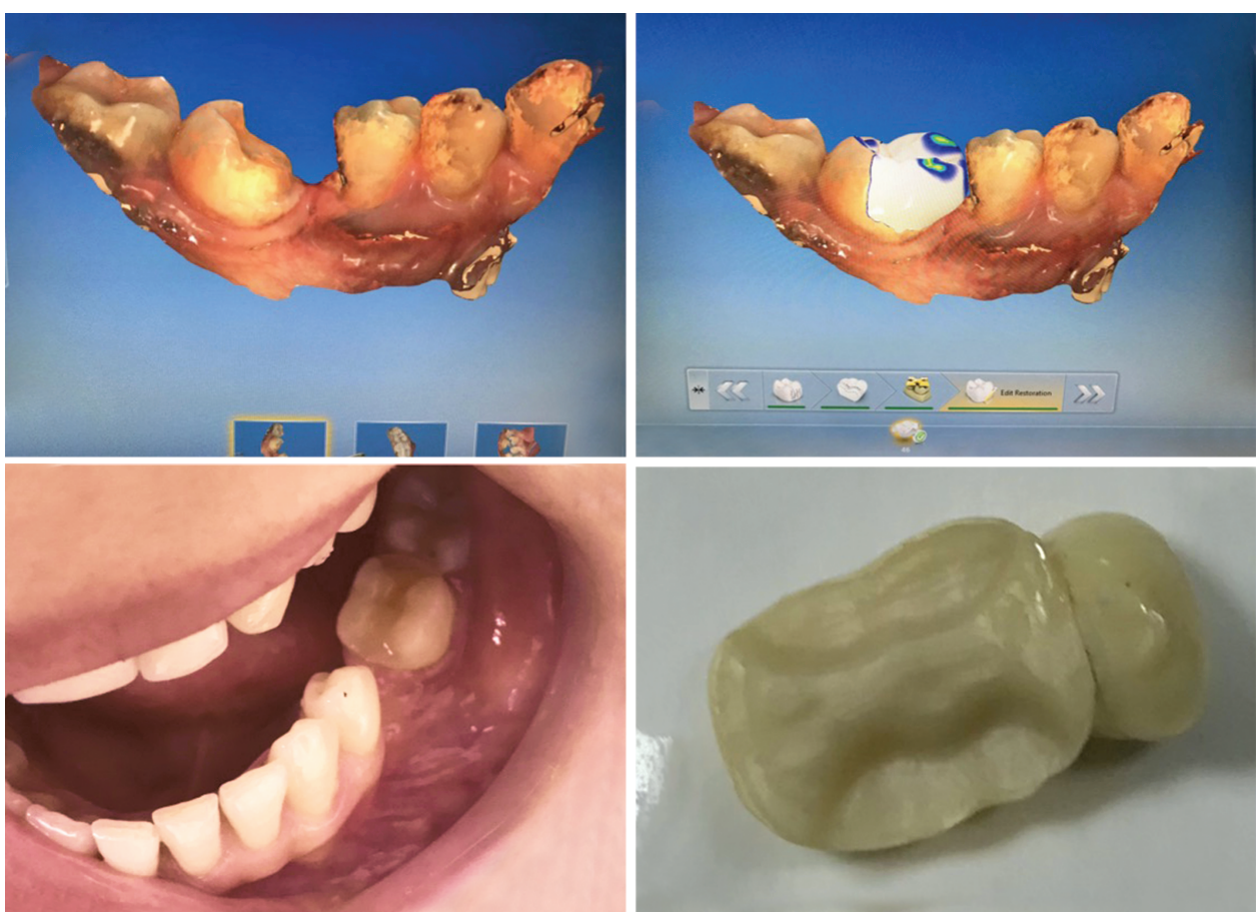

Fig. 2: Prepared abutment teeth and digital designs and milled restoration 
and adhesive resin (Single Bond Universal; 3M, Neuss, Germany) was applied and cured. Dual-cure resin cement (RelyX Ultimate Clicker; $3 \mathrm{M}$ ESPE, Neuss, Germany) was used for the cementation. Buccal sides of teeth 21, 22, and some marginal sides of tooth 37 were modified and shaped with composite resin for a better esthetic finishing (Figs 3 and 4).

During the 24 month clinical follow-up of the patient, no problem was encountered in terms of esthetic, biological, or patient satisfaction. Many laboratories and clinical follow-up studies are still needed to clinically recommend such large volume restorations.

\section{Discussion}

This case report illustrates an alternative approach to the rehabilitation of narrow edentulous spaces with ceramic materials by CAD/CAM technique with minimally invasive approaches. With the development of CAD/CAM systems in dentistry, the number of patient visits decreased and the need for temporary restoration and traditional impression procedures was eliminated. ${ }^{11}$ Along with the development of adhesive resin materials and techniques, alternative treatments have been tried with more conservative approaches. The cases of narrow posterior edentulous spaces are relatively difficult in terms of choice of treatment in dental clinics.

Although minimally invasive approaches are considered, clinicians prefer conventional restorations such as fixed dental prosthesis, as there is not enough clinical data on the amount of preparation and selection of restoration materials depending on the size of the edentulous space. Inlay-retained fixed dental prostheses have been a more conservative treatment approach than conventional prostheses in cases where implant treatment cannot be performed. ${ }^{12}$

In this case report, we emphasize the clinical success of feldspathic and monolithic zirconia restorations prepared with partial preparations of distal adjacent molar teeth in the rehabilitation of narrow mandibular posterior edentulous spaces.

It was reported that MDP-containing primer and resin cement application after the tribochemical silica coating of Y-TZP may show some advantages for the bonding strength of these materials. ${ }^{10}$ When the Y-TZP surfaces were blasted with $\mathrm{Al}_{2} \mathrm{O}_{3}$ particles, the use of a MDP-containing primer was also accepted as an important treatment for promoting a stable bond to Y-TZP. ${ }^{9}$

Furthermore, the use of MDP-containing primer and/or resin cements and moderate pressure (with a diameter between $30 \mu \mathrm{m}$ and $50 \mu \mathrm{m}$, at a pressure between 0.5 bar and 2.5 bar, duration of at least 20 seconds) sandblasting to increase the bonding strength of zirconia ceramics might be suggested. ${ }^{13}$

Sandblasting of zirconia restoration was performed and then silane was applied to these surfaces to increase the bonding
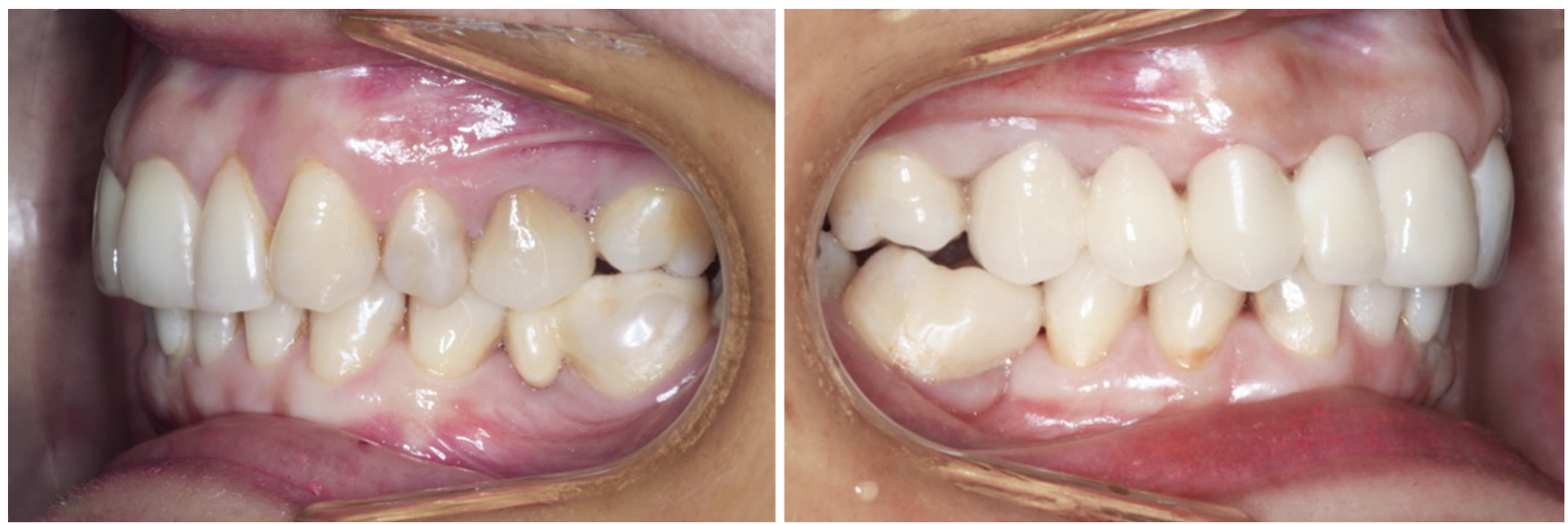

Fig. 3: Buccal views of the CAD/CAM restorations
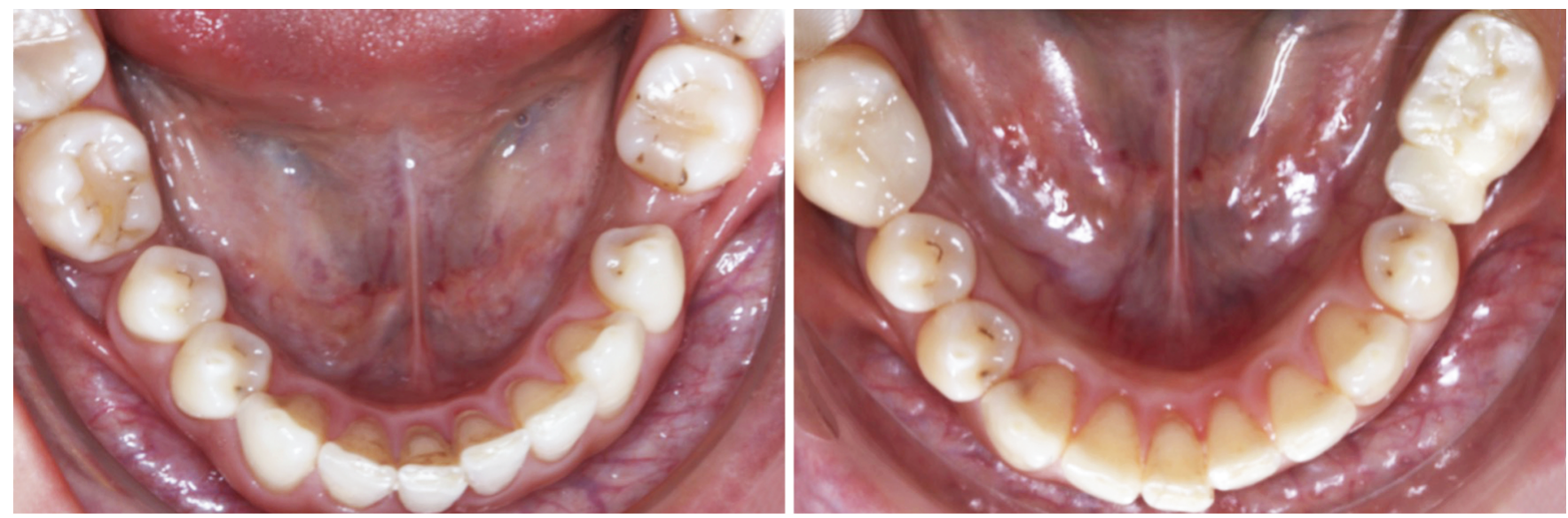

Fig. 4: Occlusal views of the CAD/CAM restorations before and after the treatment 
strength before resin cementation. Also, adhesive resin systems containing MDP, known to increase bond strength in monolithic zirconia, was used in the cementation process, which was also recommended in the literature. ${ }^{14}$

Hydrofluoric acid is one of the most widely used acids in the mechanical surface treatment of glass ceramics. Hydrofluoric acid dissolves the glassy phase and makes the surface porous. Good micromechanical retention is obtained when the resin cement penetrates into these porous surfaces. ${ }^{15,16}$

It is also known that the bond strength between resin cement and feldspathic ceramic may be increased by hydrofluoric acid and silane coupling agent surface treatments. ${ }^{17}$ Hydrofluoric acid and silane surface treatments were preferred when cementing feldspathic ceramic in this case report.

Especially in all-ceramic restorations with a zirconia substructure, the most common technical problem was minor separations or fractures in the superstructure ceramics. In the meta-analysis performed by Sailer et al., ${ }^{18}$ the detachments and fractures seen in the ceramic superstructure in all-ceramic fixed partial dentures supported by zirconia were found to be between $6.6 \%$ and $26 \%$ after 5 years. In order to prevent these complications, monolithic zirconia ceramic systems, which can be applied in one piece, have been put forward. ${ }^{19}$

Esthetic properties of zirconia ceramics have been improved in recent years. ${ }^{19,20}$ For a better shade matching, they may be colored before sintering process. ${ }^{21}$ In addition, when this might not be enough for the most desired esthetic results, the framework design might be formed for veneering the buccal surfaces of these restorations with layering ceramics. ${ }^{21,22}$

Bomicke et al. ${ }^{23}$ reported that in a three-year clinical study, monolithic and partially coated zirconia crowns may be used clinically without compromising good survival (monolithic zirconia $100 \%$, partially coated zirconia $98.5 \%$ ).

In the current case report, monolithic zirconia with a veneer design on the buccal side was used in order to achieve an esthetically satisfactory result and to minimize the risk of chipping.

It may be confusing to decide on the restorations where a narrower edentulous space exists than a tooth width. When the minimally invasive approach is preferred, onlay-retained fixed partial dentures or larger volume restorations will be discussed by using the mechanical advantages of ceramic block technology and the adhesive techniques. There were no mechanical problems in this case after 2 years' follow-up. But it is clear that there is not enough scientific data to make stronger recommendations for the limits and the long-term follow-up of these restorations.

\section{Conclusion}

The CAD/CAM restorations prepared with monolithic zirconia or feldspathic ceramics were successful in the cases of narrow posterior edentulous spaces after the second-year follow-up. Distal molar tooth-supported ceramic restorations prepared with CAD/CAM technique are promising in the rehabilitation of narrow posterior edentulous spaces.

\section{References}

1. Xie Q, Lassila LVP, Vallittu PK. Comparison of load-bearing capacity of direct resin-bonded fiber-reinforced composite FPDs with four framework designs. J Dent 2007;35(7):578-582. DOI: 10.1016/j. jdent.2007.04.003.
2. Jung RE, Zembic A, Pjetursson BE, et al. Systematic review of the survival rate and the incidence of biological, technical, and aesthetic complications of single crowns on implants reported in longitudinal studies with a mean follow-up of 5 years. Clin Oral İmplants Res 2012;23(Suppl 6):2-21. DOI: 10.1111/j.1600-0501.2012.02547.x.

3. Karaarslan ES, Ertas E, Ozsevik S, et al. Conservative approach for restoring posterior missing tooth with fiber reinforcement materials: four clinical report. Eur J Dent 2011;5(4):465-471. DOI: 10.1055/s-00391698920.

4. Russo DS, Cinelli F, Sarti C, et al. Adhesion to zirconia: a systematic review of current conditioning methods and bonding materials. Dent J 2019;7:74. DOI: 10.3390/dj7030074.

5. Schmitter M, Mueller D, Rues S. Chipping behaviour of all-ceramic crowns with zirconia framework and cad/cam manufactured veneer. J Dent 2012;40(2):154-162. DOI: 10.1016/j.jdent.2011.12.007.

6. Tinschert J, Natt G, Mohrbotter N, et al. Lifetime of alümina and zirconia ceramics used for crown and bridge restorations. J Biomed Mater Res 2007;80(2):317-321. DOI: 10.1002/jbm.b.30599.

7. Khan $A A, A I$ Kheraif $A A$, Jamaluddin $S$, et al. Recent trends in surface treatment methods for bonding composite cement to zirconia: a review. J Adhes Dent 2017;19(1):7-19.

8. Atsu SS, Kilicarslan MA, Kucukesmen HC, et al. Effect of zirconium oxide ceramic surface treatments on the bond strength to adhesive resin. J Prosthet Dent 2006;95(6):430-436. DOI: 10.1016/j. prosdent.2006.03.016.

9. Lima RBW, Barreto SC, Alfrisany NM, et al. Effect of silane and MDPbased primers on physico-chemical properties of zirconia and its bond strength to resin cement. Dent Mater 2019;35(11):1557-1567. DOI: $10.1016 /$ j.dental.2019.07.008.

10. Lim MJ, Yu MK, Lee KW. The effect of continuous application of MDP-containing primer and luting resin cement on bond strength to tribochemical silica-coated Y-TZP. Restor Dent Endod 2018;43(2):19. DOI: 10.5395/rde.2018.43.e19.

11. Palin W, Burke FJ. Trends in indirect dentistry:8.CAD/CAM technology. Dent Update 2005;32(10):566-572. DOI: 10.12968/ denu.2005.32.10.566.

12. Augusti D, Augusti G, Borgonovo A, et al. Inlay-retained fixed dental prosthesis: a clinical option using monolithic zirconia. Case Rep Dent 2014;2014:629786. DOI: 10.1155/2014/629786.

13. Kern M. Bonding to oxide ceramics-laboratory testing versus clinical outcome. Dent Mater 2015;31(1):8-14. DOI: 10.1016/ j.dental.2014.06.007.

14. Zandparsa $R$, Talua NA, Finkelman MD, et al. An in vitro comparison of shear bond strength of zirconia to enamel using different surface treatments. J Prosthodont 2014;23:117-123. DOI: 10.1111/jopr.12075.

15. Chen JH, Matsumura H, Atsuta M. Effect of etchant, etching period, and silane priming on bond strength to porcelain of composite resin. Oper Dent 1998;23(5):250-257.

16. Brentel AS, Özcan M, Valandro LF, et al. Microtensile bond strength of a resin cement to feldpathic ceramic after different etching and silanization regimens in dry and aged conditions. Dent Mater 2007;23(11):1323-1331. DOI: 10.1016/j.dental.2006.11.011.

17. Graiff $L$, Piovan $C$, Vigolo $P$, et al. Shear bond strength between feldspathic CAD/CAM ceramic and human dentine for two adhesive cements. J Prosthodont 2008;17(4):294-299. DOI: 10.1111/j.1532849X.2007.00294.x.

18. Sailer I, Pjetursson BE, Zwahlen M, et al. A systematic review of the survival and complication rates of all-ceramic and metal ceramic reconstructions after an observation period of at least 3 years. Part II: fixed dental prostheses. Clin Oral Implants Res 2007;18:86-96. DOI: 10.1111/j.1600-0501.2007.01468.x.

19. Ueda K, Guth JF, Erdelt K, et al. Light transmittance by a multi-coloured zirconia material. Dent Mater J 2015;34(3):310-314. DOI: 10.4012/ dmj.2014-238.

20. Zarone F, Di Mauro MI, Ausiello P, et al. Current status on lithium disilicate and zirconia: a narrative review. BMC Oral Health 2019;19(1):134. DOI: 10.1186/s12903-019-0838-x. 
21. Rinke S, Fischer C. Range of indications for translucent zirconia modifications: clinical and technical aspects. Quintessence Int 2013;44(8):557-566.

22. Moscovitch M. Consecutive case series of monolithic and minimally veneered zirconia restorations on teeth and implants: up to
68 months. Int J Periodontics Restorative Dent 2015;35(3):315-323. DOI: 10.11607/prd.2270.

23. Bomicke W, Rammelsberg P, Stober T, et al. Short-term prospective clinical evaluation of monolithic and partially veneered zirconia single crowns. J Esthet Restor Dent 2017;29(1):22-30. DOI: 10.1111/jerd.12270. 